\title{
Report on the 6th international workshop of the CCN family of genes
}

\author{
Stephen M. Twigg • Sandra Irvine • Andrew Leask • \\ Bernard Perbal
}

Received: 14 December 2010 / Accepted: 14 December 2010/Published online: 15 January 2011

(C) The International CCN Society 2011

The 6th International Workshop of the CCN Family of Genes was held from October 20th-24th 2010, at the grand, picturesque location of the Slieve Donard Hotel, in Newcastle, Northern Ireland, and was efficiently hosted by Dr Sandra Irvine on behalf of Queens University Belfast.

As in previous years, the Workshop programme was delivered by a mixture of speakers who were either invited or were selected based on their submitted abstracts. Following a warm opening welcome by Bernard Perbal

\section{S. M. Twigg $(\bowtie)$}

Sydney Medical School - The University of Sydney, Blackburn Building (D06), The University of Sydney, NSW 2006, Australia

e-mail: stephen.twigg@sydney.edu.au

\section{S. M. Twigg}

Department of Endocrinology,

Royal Prince Alfred Hospital Sydney,

Blackburn Building (D06), The University of Sydney,

NSW 2006, Australia

\section{S. Irvine}

Haematology Department, Centre for Cancer Research and Cell Biology, Queen's University Belfast,

97, Lisburn Rd,

BT9 7BL, Belfast, UK

e-mail: s.irvine@qub.ac.uk

\section{A. Leask}

Division of Oral Biology, Schulich School of Medicine and Dentistry, University of Western Ontario,

London, ON N6A 5C1, Canada

e-mail: Andrew.Leask@schulich.uwo.ca

\section{B. Perbal}

L' Oréal Recherche et Innovation,

Direction des Sciences du Vivant,

Clichy 92583, France

e-mail: BPERBAL@rd.loreal.com
(L'Oréal Recherche and Innovation, Clichy, France) and Sandra Irvine (Queen's University, Belfast, Northern Ireland) on the evening of October 20th, it commenced with a Keynote lecture by James E. Trosko (Michigan State University, East Lansing, USA) in a 'tour de force' addressing the role of cell-cell communications through gap junctions. Whereas it was disappointing that, due to pneumonia, the initial Keynote presenter, ICCNS Awardee Prof. Edward Calabrese (University of Massachusetts, Amherst, USA), was unable to attend the Workshop and deliver his presentation on hormesis, J. Trosko challenged the audience and current paradigms in scientific thought.

$J$. Trosko presented evidence that such junctions are integral to stem cell and differentiated cell homeostatic functions. He further proposed that gap junctions may be an under-appreciated target to prevent and treat human disease including in common epithelial malignancies where cancer stem cells may contribute to cancer pathogenesis.

A series of other invited educational talks were delivered across subsequent days. A keynote lecture delivered by Richard Kendall from the human therapeutics company, AMGEN (Thousand Oaks, CA, USA), described the progress in the field of angiogenic inhibitors. He indicated that such therapies have demonstrated clear benefit in a variety of in vitro and preclinical models of angiogenesis, and have shown promise in a number of human clinical cancer trials. Agents under study include AMG 386, a peptibody that binds to and inhibits angiopoietins 1 and 2 in the angiopoietin/Tie2 pathway, and inhibition of the VEGF/ VEGFR pathway with AMG273. It is envisaged that as knowledge of angiogenesis regulation improves, more specific regulators of angiogenesis including their use in combination will improve outcomes in those who develop angiogenesis dependent human disease, such as many epithelial malignancies. 
Further symposia sessions were delivered by invited speakers. Thought provoking topics included Hormesis by C. Mothersill (McMaster University, Hamilton, Ontario, Canada) and L.E Feinendegen (Heinrich-Heine-University, Düsseldorf, Germany) who each focused on non-linear cell and tissue effects of radiation exposure; and angiogensis as a target coming undone, by A. Reynolds (John Vane Science Centre, Charterhouse Square, London UK). The symposium on Inflammation was addressed by five diverse presentations: Natural compounds from marine products by M. Diederich (Hôpital Kirchberg, Luxembourg); Dissociating effects of glucocorticoids by G. Haegeman (Ghent University, Gent, Belgium); Novel regulators of proinflammation including cannabinoids by P. Moynagh (National University of Ireland, Maynooth, Ireland); Cox-2 inhibitors by C. Cerella (Hôpital Kirchberg, Luxembourg); and TNF$\alpha$ interaction effects across death and non-death pathways by E. Dejardin (Katholieke Universiteit Leuven and Universiteit Hasselt, Leuven, Belgium). A special presentation was also made by L. Micutkova (Austrian Academy of Sciences, Innsbruck, Austria), examining IGFBP-3 nuclear uptake and comparisons with $\mathrm{CCN}$ proteins.

The academic program of oral communications, selected from submitted abstracts, on the $\mathrm{CCN}$ family of genes dominated the meeting. Each of the CCN genes - from CCN1 to CCN6 were addressed across the Workshop.

For CCN1, K. Sumiyoshi (Okayama University Graduate School of Medicine, Dentistry and Pharmaceutical Sciences, Okayama, Japan) described its regulation through the 3'UTR untranslated region and its related biological significance. $T$. Quan (University of Michigan, Ann Arbor, USA) showed that knockdown of CYR61 significantly attenuates UV irradiationinduced inhibition of type-I procollagen and upregulation of MMP-1 in skin fibroblasts; CCN1 was up-regulated by UV irradiation through a transcription factor AP-1 binding site to induce type 1 Collagen, and it was inhibited by retinoids in skin, the latter which suppress its gene expression and protein levels with implications for ageing in vivo.

In terms of $\mathrm{CCN} 1$ post-translational regulation, $A$. Guillon-Munos (Université François-Rabelais de Tours, France) reported that the serine protease Kallikrein related peptidase- 12 potently hydrolyses $\mathrm{CCN} 1$ to convert it into biologically relevant protein split products; this enzyme also has activity on other CCN1 family members. In terms of CCN1 bioactivity, this protein was reported by A. Sabile (ETH-Hönggerberg, Zurich, Switzerland) to promote in vivo tumour growth in a mouse model of osteosarcoma, suggesting that it may be a therapeutic target in this setting.

As in previous International CCN Workshops, presentations into CCN2 were most prominent across the sessions. This may be because CCN2 continues to be implicated in many diseases involving fibrosis and also types of cancer. Progress continues to be made into $\mathrm{CCN} 2$ regulation. $P$.
Trackman (Henry M. Goldman School of Dental Medicine, Boston, USA) reported that $\mathrm{CCN} 2$ is regulated in a cell and tissue specific manner in that human gingival fibroblasts are affected by GSK $3 \beta$ in a differential manner compared with human lung fibroblasts; this work suggests that specific targets in a topical oral mucosal application against CCN2 may be able to be developed to treat for example, conditions of gingival overgrowth. Also in $\mathrm{CCN} 2$ regulation, A. Thomas-Tikhonenko and his student J. Fox (University of Pennsylvania Philadelphia, USA) presented work indicating that specific microRNAs, of the 17-92 cluster, are important regulators of CCN2, including in glioblastoma. As microRNAs are master regulators it follows that their targeting may become a method of treating disease and in health maintenance.

In addressing CCN2 action, a series of presentations added further complexity and challenge to the field. $E$. Aoyama (Okayama University Graduate School of Medicine, Dentistry and Pharmaceutical Sciences) reported in a robust series of experiments that $\mathrm{CCN} 2$ binds to the FGF2R and modulates its signalling in vitro and in vivo including in chondrocytes. CCN2 new binding partners reported include matrilyn-3 as reported by $T$. Hattori (Okayama University Graduate School of Medicine, Dentistry and Pharmaceutical Sciences) and RANK ligand, shown by S. Kubota (Okayama University Graduate School of Medicine, Dentistry and Pharmaceutical Sciences); the physiological relevance of these respective findings and implications for human disease including in bone and cartilage, will be followed with much interest.

CCN2 physiological effects are being reported in increasingly diverse cell types including mesenchymal stem cells and pulmonary cells by $R$. Laug (University of Würzburg, Germany) and in mediating fat cell differentiation as described by S. Twigg (University of Sydney, Australia).

$\mathrm{CCN} 2$ in pathological states was also presented. In a series of elegant experiments detailed by A. Leask (University of Western Ontario, London, Canada), CCN2 was shown to be derived from pericyte cells in skin in the model of bleomycin induced scleroderma. CCN2 was also implicated by B. Riser (Rosalind Franklin University of Medicine and Science, Chicago and Baxter, USA) in the recently described condition of gadolinium induced nephrogenic induced systemic fibrosis. A body of work from $E$. Brandan (Pontificia Universidad Católica de Chile, Santiago, Chile) showed that CCN2 may be useful target to prevent Duchenne muscular dystrophy, and S. Twigg (University of Sydney, Australia) indicated that a rodent model of diabetes exacerbating NASH fibrosis induces CCN2 mRNA and protein in hepatic cells.

In contrast, $\mathrm{CCN} 2$ was impressively shown to protect against age-related osteoarthritis by S. Itoh (Okayama 
University Graduate School of Medicine, Dentistry and Pharmaceutical Sciences) when it was over-expressed in cartilage in mice. Finally, in a series of complementary studies, Ahmed, Attramadal and Martinov (University of Oslo, Norway) reported that high concentrations of rhCCN2 in vitro and high levels of its expression in vivo in cardiac cells are cardioprotective through activation of the PI3K-Akt/PKB-GSK3 $\beta$ signalling pathway.

For CCN3, most of the communications examined its bioactivities. $\mathrm{CCN} 3$ holds much promise as an agent to augment current therapy in chronic myeloid leukaemia as shown by L. McCallum (Queen's University, Belfast, Northern Ireland), a condition where as reported by $S$. Suresh (Queen's University, Belfast, Northern Ireland), CCN3 is regulated by specific microRNAs. In vascular studies in vivo K. Yokote (Chiba University Graduate School of Medicine, Chiba, Japan) showed that CCN3 prevented development of neointimal hyperplasia. Finally, CCN3 was shown to induce neural outgrowth by $W-C$. Sin, and like CCN2, B. Riser (Rosalind Franklin University of Medicine and Science, Chicago and Baxter, USA) implicated it in gadolinium induced nephrogenic induced systemic fibrosis, where on the basis of renal fibrosis studies in the diabetic kidney, CCN3 would be predicated to be counter-regulatory and to inhibit $\mathrm{CCN} 2$ and the fibrogenesis.

Regarding CCN4, the main focus was on bone and cartilage development, where CCN4 function in stem cells and chondrocytes was described by K. Schlegelmilch (University of Würzburg, Germany) and CCN4 was shown by M. Ono (National Institutes of Craniofacial and Dental Research, Bethesda,USA) to regulate osteogenesis by enhancing BMP-2 activity.

For CCN5 its regulation and action in vascular smooth muscle cells was presented by $K$. Wiesman (Tufts University, Boston, USA).

CCN6 also had limited representation at the Workshop, where it was implicated by C. Kleer (University of Michigan, Ann Arbor, USA) in breast cancer cell invasive phenotype.

In summary, across the $\mathrm{CCN}$ genes and proteins family, broad topic areas included $\mathrm{CCN}$ regulation, $\mathrm{CCNs}$ action and interaction, and the roles of CCNs in physiology and pathology. A small number of studies across the $\mathrm{CCN}$ genes and proteins compared and contrasted findings in physiology and pathology, notably B. Riser (Rosalind Franklin University of Medicine and Science, Chicago and Baxter, USA) in $\mathrm{CCN} 2$ and $\mathrm{CCN} 3$ regulation in gadolinium induced nephrogenic induced systemic fibrosis, and A. Guillon-Munos (Université François-Rabelais de
Tours, Tours, France) who determined that Kallikrein related peptidase- 12 potently hydrolyses both $\mathrm{CCN} 1$ and CCN5 preferentially to other CCN proteins.

With thanks to Springer for sponsoring our Young Springer Awardees: J. Fox (University of Pennsylvania, Philadelphia, USA), S. Itoh (Okayama University Graduate School of Medicine, Dentistry and Pharmaceutical Sciences) and K. Wiesman (Tufts University, Boston, USA), each of whom presented entertaining talks as indicated above. A small series of posters also featured and were informally discussed at allocated poster sessions. The social program which included dinner events at the Stormont Parliament Buildings, and a Gala Dinner and dance with an Irish band at Slieve Donard, were well received and assisted informal meetings and planning of future experimental collaborations among attendees.

Overall, this 6th Worskhop was felt to be highly successful considering the diversity of material presented, the level of interaction between attendees during the meeting, and its excellent organisation. Nearly all continents of the world were represented by the 70 or more registrants. While it was disappointing that some of our colleagues including founders and members of the International CCN Society did not attend the event, it is already planned that a number of North American colleagues will present at an ICCNsponsored meeting focused on Connective Tissue Disease in Cancer in 2011 in Vancouver, BC, Canada (see http:// www.ccnsociety.com for information).

The7th International CCN Workshop will be held in 2012 in Sydney, Australia.

In summary, the meeting emphasized the increasing evidence that the CCN family plays an essential role in physiology; regulation of $\mathrm{CCN}$ genes and proteins may have clinical value in modifying the outcome of pathological states. Further information about $\mathrm{CCN}$ gene regulation including by microRNAs, at the level of mRNA stability and $\mathrm{CCN}$ protein post-translational regulation including by common mechanisms for example by families of proteases will undoubtedly be generated as the field evolves. At present, blocking CCN2 for example in diabetic nephropathy and in idiopathic interstitial lung disease using humanised antibodies holds promise, and $\mathrm{CCN} 3$ as adjunct therapy in CML may ultimately prove to have utility. Measuring the relative abundance of $\mathrm{CCN}$ proteins may also have value in defining disease or predicting disease course.

The future for $\mathrm{CCN}$ proteins in human health and disease appears bright and it is expected that interest in the field will continue to intensify. 